

\section{DISCLAIMER}

This report was prepared as an account of work sponsored by an agency of the United States Government. Neither the United States Government nor any agency Thereof, nor any of their employees, makes any warranty, express or implied, or assumes any legal liability or responsibility for the accuracy, completeness, or usefulness of any information, apparatus, product, or process disclosed, or represents that its use would not infringe privately owned rights. Reference herein to any specific commercial product, process, or service by trade name, trademark, manufacturer, or otherwise does not necessarily constitute or imply its endorsement, recommendation, or favoring by the United States Government or any agency thereof. The views and opinions of authors expressed herein do not necessarily state or reflect those of the United States Government or any agency thereof. 


\section{DISCLAIMER}

Portions of this document may be illegible in electronic image products. Images are produced from the best available original document. 


\section{NOTICE}

This report was prepared as an account of work sponsored by the United State Government. Neither the United States nor the United States Energy Research and Development Administration, nor any of their employees, nor any of their contractors, subcontractors, or their employees, makes any warranty, express or

implied, or assumes any legal liability or responsibility for the accuracy, com-

pleteness or usefulness of any information, apparatus, product or process disclosed or represents that its use would not infringe privately owned rights. 


\title{
Program for Calculating X-Ray Powder Diffraction Interplanar (d) Spacings with a Tektronix-31 Desk Top Programmable Calculator
}

\author{
Ralph R. Eckstein and Yoshio G. Ishida
}

Issued: February 22, 1977

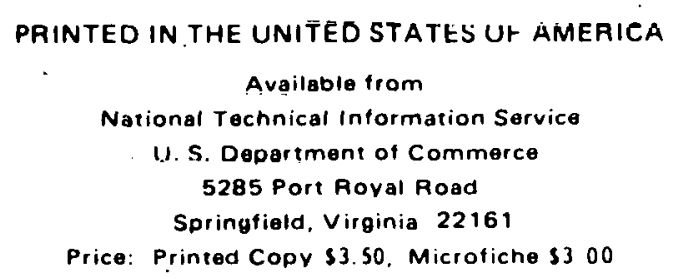

MONSANTO RESEARCH CORPORATION

A Subsidiary of Monsanto Company

\section{mOUnD LABORATORY}

Miamisburg, Ohio

45342

operated for

\section{UNITED STATES ENERGY RESEARCH AND DEVELOPMENT ADMINISTRATION}

U. S. Government Contract No. EY-76-C-04-0053 


\begin{abstract}
A Tektronix-31 (Tektronix Inc. Beaverton, Oregon) desk top calculator program, which is used as a backup to the PDP-8/I computer program, is described. The program yields interplanar (d) spacings and $(2 \theta)$ angle values from. measurements made on an X-ray diffraction film of a powdered sample of a. crystalline material. Use of the calculator provides accurate computations in a relatively rapid time interval when the PDP-8/I is not functioning because of downtime for repairs, maintenance, etc.
\end{abstract}




\section{Introduction}

This report relates the capabilities of the Tektronix-31 desk top calculator when used as a backup for the PDP-8/I computer for obtaining accurate and relatively rapid data computations. A program has been written to process line measurements obtained from X-ray powder diffraction films. Essentially, this program is identical in its structure to the one written for use with the PDP-8/I in that it yields interplanar spacings (d-spacings), diffraction angle theta values $(2 \theta)$, and the camera radius correction factor. Although the

Tektronix-31 does compute the data as accurately as does the PDP-8/I, its major shortcomings lie in two areas: the I/O (input/output) of data is slower by a factor of four, and the data presentation is rather bulky and cumbersome to handle. These areas are readily acceptable, however, when hand calculations are the alternative since the computation of about-40 diffraction lines can be accomplished using the Tektronix31 calculator in approximately $10 \mathrm{~min}$, whereas hand calculations would require on the order of $4 \mathrm{hr}$.

The Tektronix-3l has the additional advantage of being capable of transferring data from its memory registers to a tape cassette. This is not only desirable to retain programs for future use; but it is very desirable when the program, as described in this report, is considered. This program, written for $X$-ray data computation, is composed of 1088 instructions and would be extremely tedious to enter each time it were used without the cassette tape capability.

\section{Mathematical Equations}

As in:the program written for the PDP$8 / I$, the equation of prime importance in determining interplanar spacings (a-spacings) dnd lie diffraction anglc theta values is the Bragg equation:

$$
\lambda=2 \mathrm{~d} \sin \theta
$$

\author{
where: $\lambda=$ wavelength of copper radia- \\ tion used $(K \bar{\alpha}=1.54178 \AA$, \\ $\left.\mathrm{K} \alpha_{1}=1.54051 \AA, \mathrm{K} \alpha_{2}=1.54433,\right)$ \\ $\mathrm{d}=$ interplanar spacing \\ $2 \theta=$ angle which describes the \\ deviation of diffracted X-rays \\ from the direct beam.
}

\section{Discussion}

This report contains a copy of the machine language program that was written to process a maximum of $60 \mathrm{x}$-ray powder diffraction line measurements. With some modifications of the $R$ registers used, ${ }^{2}$ additional lines could be adequately handled. However, a pattern containing more than 60 different lines is not a common occurrence, As with the use of the PDP-8/I program when the data for more than 60 lines is desired, the input can be divided into two segments and thus adequately handled. Figure 1 . shows the program that will routinely process 60 lines of a powder diffraction pattern. Figure 2 shows a simplified version of the flow chart. Figure 3 (Sections A, B, C, and D) shows the flow chart which is outlined prior to writing the program.

The Tektronix-3l calculator has a maximum of $640 \mathrm{R}$ storage registers plus an additiona $\bar{l}-10$ of $K$-registers. The $R$ registers were not used. ${ }^{-}$As was pre=viously stated, all of the $R$.. and $K$ registers have not been utilizè; and with careful rearrangement, additional storage of data and the computational capabilities of the calculator could be significantly increased.

Presently, the first $360 \mathrm{R}$ - registers are reserved for data input $\overline{\text { storage. }}$ There are $60 \mathrm{R}$. registers reserved to store information about the left line, 


\begin{tabular}{|c|c|c|c|c|c|c|c|c|c|c|c|}
\hline 0000 & EXC_ & 0063 & CLDP & $\begin{array}{l}0126 \\
0127\end{array}$ & $\begin{array}{l}\text { IF_0 } \\
\text { EX } \bar{C}_{-}\end{array}$ & $\begin{array}{l}0190 \\
0191\end{array}$ & $\begin{array}{l}0 \\
\text { PRNT }\end{array}$ & $\begin{array}{l}0254 \\
0255\end{array}$ & $\begin{array}{l}4 \\
1\end{array}$ & $\begin{array}{l}0318 \\
0319\end{array}$ & $\begin{array}{l}= \\
\mathrm{R}\end{array}$ \\
\hline 0001 & $\mathrm{ARC}^{-}$ & 0064 & 1 & 0128 & & 0192 & $\mathrm{EXC}$ & 0256 & CLDP & 0320 & \\
\hline 0002 & LBL & 0065 & $=$ & 0129 & CONT & 0193 & & 0257 & +- & 0321 & 4 \\
\hline 0003 & $\mathrm{DG} / \overline{\mathrm{R}}$ & 0066 & $R_{-}$ & 0130 & L & 0194 & B & 0258 & $=$ & 0322 & 6 \\
\hline 0004 & CLDP & 0067 & & 0131 & ( & 0195 & 1 & 0259 & 6 & 0323 & CLDP \\
\hline 0005 & P & 0068 & 0 & 0132 & I & 0196 & I & 0260 & 4 & 0324 & 1 \\
\hline 0006 & R & 0069 & 0 & 0133 & ) & 0197 & ) & 0261 & 5 & 0325 & $=$ \\
\hline 0007 & 0 & 0070 & $=$ & 0134 & $=$ & 0198 & $=$ & 0262 & 9 & 0326 & 5 \\
\hline 0008 & G & 0071 & $\mathrm{~K}$ & 0135 & CLDP & 0199 & CLDP & 0263 & 6 & 0327 & 4 \\
\hline 0009 & $\mathrm{R}$ & 0072 & $3^{-}$ & 0136 & STOP & 0200 & STOP & 0264 & 4 & 0328 & 0 \\
\hline 0010 & A & 0073 & $=$ & 0137 & $=$ & 0201 & $=$ & 0265 & $=$ & 0329 & 5 \\
\hline 0011 & M & 0074 & $R_{---}$ & 0138 & R & 0202 & $\mathrm{R}$ & 0266 & $\mathbf{R}$ & 0330 & 1 \\
\hline 0012 & & 0075 & & 0139 & & 0203 & & 0267 & & 0331 & $=$ \\
\hline 0013 & $\mathrm{~T}$ & 0076 & 3 & 0140 & & 0204 & $0^{---}$ & 0268 & 4 & 0332 & $\mathbf{R}$ \\
\hline 0014 & 0 & 0077 & 1 & 0141 & 0 & 0205 & 0 & 0269 & 2 & 0333 & 4 \\
\hline 0015 & & 0078 & CLDP & 0142 & 0 & 0206 & 0 & 0270 & CLDP & 0334 & 4 \\
\hline 0016 & C & 0079 & 2 & 0143 & PRNT & 0207 & PRNT & 0271 & $=$ & 0335 & 7 \\
\hline 0017 & A & 0080 & $=$ & 0144 & $\mathrm{EXC}$ & 0208 & EXC_ & 0272 & 0 & 0336 & CLDP \\
\hline 0018 & L & 0081 & $R_{-\ldots}$ & 0145 & 4 & 0209 & 4 & 0273 & 7 & 0337 & 1 \\
\hline 0019 & C & 0082 & & 0146 & M & 0210 & $I$ & 0274 & 9 & 0338 & $=$ \\
\hline 0020 & & 0083 & 3 & 0147 & ( & 0211 & 1 & 0275 & 6 & 0339 & 5 \\
\hline 0021 & PAPR & 0084 & 2 & 0148 & I & 0212 & $I$ & 0276 & 8 & 0340 & 4 \\
\hline 0022 & D & 0085 & CLDP & 0149 & ) & 0213 & 1 & 0277 & 9 & 0341 & 4 \\
\hline 0023 & & 0086 & 3 & 0150 & $=$ & 0214 & $=$ & 0278 & $=$ & 0342 & 3 \\
\hline 0024 & $\bar{S}$ & 0087 & $=$ & 0151 & CLDP & 0215 & CLDP & 0279 & $\mathbf{R}$ & 0343 & 3 \\
\hline 0025 & $\mathrm{P}$ & 0088 & $\mathbf{R}$ & 0152 & STOP & 0216 & STOP & 0280 & 4 & 0344 & $=$ \\
\hline 0026 & A & 0089 & 4 & 0153 & $=$ & 0217 & $=$ & 0281 & 4 & 0345 & $\mathrm{R}$ \\
\hline 0027 & C & 0090 & 3 & 0154 & $\mathbf{R}$ & 0218 & $\mathrm{R}$ & 0282 & 3 & 0346 & 4 \\
\hline 0028 & I & 0091 & 3 & 0155 & & 0219 & $\mathrm{R}^{---}$ & 0283 & CLDP & 0347 & 4 \\
\hline 0029 & $\mathrm{~N}$ & 0092 & CLDP & 0156 & 0 & 0220 & $0^{---}$ & 0284 & $+1-$ & 0348 & 8 \\
\hline 0030 & G & 0093 & 4 & 0157 & 0 & 0221 & 0 & 0285 & $=$ & 0349 & CLDP \\
\hline 0031 & $\mathrm{~S}$ & 0094 & $=$ & 0158 & 0 & 0222 & 0 & 0286 & 0 & 0350 & $=$ \\
\hline 0032 & & 0095 & $\mathbf{R}$ & 0159 & PRNT & 0223 & PRNT & 0287 & 0 & 0351 & $\mathbf{R}$ \\
\hline 0033 & & 0096 & & 0160 & EXC_ & 0224 & $\mathrm{EXC}$ & 0288 & 4 & 0352 & 4 \\
\hline 0034 & PAPR & 0097 & 3 & 0161 & 4 & 0225 & 4 & 0289 & 6 & 0353 & 5 \\
\hline 0035 & PAPR & 0098 & 4 & 0162 & $\mathbf{R}$ & 0226 & EXC & 0290 & 7 & 0354 & 0 \\
\hline 0036 & $E$ & 0099 & CLDP & 0163 & ( & 0227 & HYP $^{-}$ & 0291 & 4 & 0355 & $=$ \\
\hline 0037 & $\mathrm{~N}$ & 0100 & 5 & 0164 & I & 0228 & LBL & 0292 & $=$ & 0356 & $\mathbf{R}$ \\
\hline 0038 & $\mathrm{~T}$ & 0101 & $=$ & 0165 & ) & 0229 & $\mathrm{ARC}^{-}$ & 0293 & $\mathbf{R}$ & 0357 & 4 \\
\hline 0039 & $E$ & 01.02 & & 0166 & $=$ & 0230 & 1 & 0294 & 4 & 0358 & 5 \\
\hline 0040 & R & 0103 & & 0167 & CLDP & 0231 & $=$ & 0295 & 4 & 0359 & 1 \\
\hline 0041 & & 0104 & 3 & 0168 & STOP & 0232 & 5 & 0296 & 4 & 0360 & $=$ \\
\hline 0042 & $\mathrm{~N}$ & 0105 & 5 & 0169 & $=$ & 0233 & 4 & 0297 & CLDP & 0361 & $\mathbf{R}$ \\
\hline 0043 & $=$ & 0106 & CLDP & 0170 & $\mathbf{R}$ & 0234 & 1 & 0298 & $=$ & 0362 & 4 \\
\hline 0044 & CLDP & 0107 & 6 & 0171 & & 0235 & 7 & 0299 & 0 & 0363 & 5 \\
\hline 0045 & $=$ & 0108 & $=$ & 0172 & $0^{-}$ & 0236 & 8 & 0300 & 0 & 0364 & 2 \\
\hline 0046 & $\mathrm{~K}$ & 0109 & $\mathbf{R}$ & 0173 & 0 & 0237 & $=$ & 0301 & 0 & 0365 & $=$ \\
\hline 0047 & $2^{-}$ & 0110 & $4^{---}$ & 0174 & 0 & 0238 & $\mathbf{R}$ & 0302 & 1 & 0366 & $\mathrm{R}$ \\
\hline 0048 & CLDP & 0111 & 3 & 0175 & PRNT & 0239 & & 0303 & 5 & 0367 & 4 \\
\hline 0049 & 3 & 0112 & 6 & 0176 & EXC & 0240 & 4 & 0304 & 1 & 0368 & 5 \\
\hline 0050 & 6 & 0113 & EXC & 0177 & & 0241 & 0 & 0305 & $=$ & 0369 & 3 \\
\hline 0051 & 5 & 0114 & & 0178 & C & 0242 & CLDP & 0306 & & 0370 & $\mathrm{EXC}$ \\
\hline 0052 & $=$ & 0115 & LBL_ & 0179 & ( & 0243 & 1 & 0307 & & 0371 & $\mathrm{DG} / \overline{\mathrm{R}}$ \\
\hline 0053 & & 0116 & & 0180 & I & 0244 & $=$ & 0308 & 4 & 0372 & LBL \\
\hline 0054 & & 0117 & $38 \mathrm{M} 2$ & 0181 & ) & 0245 & 5 & 0309 & 5 & 0373 & HYP \\
\hline 0055 & 6 & 0118 & $\mathrm{~K}$ & 0182 & $=$ & 0246 & 7 & 0310 & CLDP & 0374 & $\mathbf{R}$ \\
\hline 0056 & & 0119 & $2^{-}$ & 0183 & CLDP & 0247 & 0 & 0311 & 3 & 0375 & $\mathrm{R}^{-}$ \\
\hline 0057 & CLDP & 0120 & - & 0184 & STOP & 0248 & 7 & 0312 & $=$ & 0376 & 4 \\
\hline 0058 & STOP & 0121 & $\mathbf{K}$ & 0185 & $=$ & 0249 & 9 & 0313 & 1 & 0377 & 3 \\
\hline 0059 & PRNT & 0122 & $9^{-}$ & 0186 & & 0250 & 6 & 0314 & 4 & 0378 & 1 \\
\hline 0060 & $=$ & 0123 & - & 0187 & & 0251 & $=$ & 0315 & 1 & 0379 & - \\
\hline 0061 & K & 0124 & 1 & 0188 & & 0252 & $\mathrm{R}$ & 0316 & 5 & 0380 & 2 \\
\hline 0062 & $9^{-}$ & 0125 & ) & 0189 & 0 & 0253 & & 0317 & 9 & 0381 & ) \\
\hline
\end{tabular}

FIGURE 1 - Tektronix-3l program to calculate XRD d-spacings. 


\begin{tabular}{|c|c|c|c|c|c|c|c|c|c|c|c|}
\hline 0382 & $I F-0$ & 0446 & R & 0510 & R & 0574 & 4 & 0638 & 5 & 0702 & 0 \\
\hline 0383 & EXC & 0447 & 4 & 0511 & & 0575 & 5 & 0639 & 0 & 0703 & 0 \\
\hline 0384 & SUMI & 0448 & 3 & 0512 & 3 & 0576 & 0 & 0640 & 1 & 0704 & 0 \\
\hline 0385 & CONT & 0449 & 1 & 0513 & 4 & 0577 & $\mathrm{EXC}$ & 0641 & $\mathrm{R}$ & 0705 & 0 \\
\hline 0386 & $\mathrm{EXC}_{-}$ & 0450 & $\mathrm{R}$ & 0514 & - & 0578 & $\operatorname{SIN}^{-}$ & 0642 & 4 & 0706 & 1 \\
\hline 0387 & $\bar{Q}$ & 0451 & 4 & 0515 & 1 & 0579 & LBL_ & 0643 & 5 & 0707 & $=$ \\
\hline 0388 & $\mathrm{LBL}_{-}$ & 0452 & 3 & 0516 & 1 & 0580 & 3 & 0644 & 2 & 0708 & $\mathrm{R}$ \\
\hline 0389 & SUMl & 0453 & 2 & 0517 & $I F=0$ & 0581 & $\mathbf{R}$ & 0645 & $=$ & 0709 & 4 \\
\hline 0390 & 2 & 0454 & + & 0518 & EXC_ & 0582 & 4 & 0646 & $\mathrm{R}$ & 0710 & 7 \\
\hline 0391 & $=$ & 0455 & 6 & 0519 & $\cos ^{-}$ & 0583 & 5 & 0647 & & 0711 & 4 \\
\hline 0392 & $\mathrm{R}_{2}$ & 0456 & $=$ & 0520 & CONT & 0584 & 3 & 0648 & 5 & 0712 & EXC \\
\hline 0393 & $\mathrm{R}$ & 0457 & $\mathrm{R}$ & 0521 & EXC & 0585 & + & 0649 & 0 & 0713 & $\overline{\mathrm{K}}$ \\
\hline 0394 & 3 & 0458 & 4 & 0522 & $\operatorname{SIN}^{-}$ & 0586 & 1 & 0650 & EXC & 0714 & CONT \\
\hline 0395 & 6 & 0459 & 3 & 0523 & LBL_ & 0587 & $=$ & 0651 & 6 & 0715 & CLDP \\
\hline 0396 & 5 & 0460 & 2 & 0524 & $\cos$ & 0588 & $\mathbf{R}$ & 0652 & LBL_ & 0716 & $I$ \\
\hline 0397 & EXC_ & 0461 & $\mathrm{R}$ & 0525 & $R_{-}$ & 0589 & 4 & 0653 & 6 & 0717 & 8 \\
\hline 0398 & TAN & 0462 & 4 & 0526 & $\mathrm{R}$ & 0590 & 5 & 0654 & R & 0718 & 0 \\
\hline 0399 & LBL_ & 0463 & 3 & 0527 & & 0591 & 3 & 0655 & & 0719 & 1 \\
\hline 0400 & & 0464 & 3 & 0528 & 3 & 0592 & $\mathrm{R}_{-}$ & 0656 & 5 & 0720 & R \\
\hline 0401 & $R_{\ldots}$ & 0465 & + & 0529 & 5 & 0593 & 4 & 0657 & 1 & 0721 & 4 \\
\hline 0402 & $\mathrm{R}_{---}$ & 0466 & 6 & 0530 & - & 0594 & 5 & 0658 & I & 0722 & 7 \\
\hline 0403 & & 0467 & $=$ & 0531 & 1 & 0595 & 1 & 0659 & $\mathrm{R}$ & 0723 & 2 \\
\hline 0404 & 3 & 0468 & $\mathbf{R}$ & 0532 & 1 & 0596 & + & 0660 & & 0724 & $=$ \\
\hline 0405 & 1 & 0469 & & 0533 & $I F=0$ & 0597 & ( & 0661 & 5 & 0725 & $\mathrm{R}$ \\
\hline 0406 & - & 0470 & 3 & 0534 & EXC_ & 0598 & R & 0662 & 3 & 0726 & 4 \\
\hline 0407 & 1 & 0471 & 3 & 0535 & 3 & 0599 & $R_{-}$ & 0663 & $=$ & 0727 & 7 \\
\hline 0408 & ) & 0472 & $\mathbf{R}$ & 0536 & CONT & 0600 & 4 & 0664 & $\mathrm{R}_{-}$ & 0728 & 4 \\
\hline 0409 & $I F=0$ & 0473 & 4 & 0537 & EXC_ & 0601 & 3 & 0665 & & 0729 & PRNT \\
\hline 0410 & EXC_ & 0474 & 3 & 0538 & 2 & 0602 & 2 & 0666 & 5 & 0730 & EXC_ \\
\hline 0411 & SUMŌ & 0475 & 4 & 0539 & LBL & 0603 & + & 0667 & 1 & 0731 & $\overline{\mathrm{K}}$ \\
\hline 0412 & CONT & 0476 & + & 0540 & 2 & 0604 & R & 0668 & $\mathrm{R}$ & 0732 & LBL \\
\hline 0413 & $\mathrm{EXC}$ & 0477 & 6 & 0541 & $\mathrm{R}$ & 0605 & $\mathrm{R}_{-}^{-}$ & 0669 & 4 & 0733 & $\overline{\mathrm{k}}$ \\
\hline 0414 & TAN $^{-}$ & 0478 & $=$ & 0542 & 4 & 0606 & 4 & 0670 & 5 & 0734 & PAPR \\
\hline 0415 & $\mathrm{LBL}_{-}$ & 0479 & R & 0543 & 5 & 0607 & 3 & 0671 & 1 & 0735 & PAPR \\
\hline 0416 & SUMŌ & 0480 & 4 & 0544 & 2 & 0608 & 3 & 0672 & - & 0736 & PAPR \\
\hline 0417 & 1 & 0481 & 3 & 0545 & + & 0609 & ) & 0673 & $R_{-}$ & 0737 & L \\
\hline 0418 & $=$ & 0482 & 4 & 0546 & 1 & 0610 & I & 0674 & 4 & 0738 & 0 \\
\hline 0419 & $R_{-}$ & 0483 & $\mathrm{R}$ & 0517 & $=$ & 0611 & 2 & 0675 & 5 & 0739 & $\mathrm{G}$ \\
\hline 0420 & $\mathbf{R}_{-}$ & 0484 & & 0548 & R & 0612 & $=$ & 0676 & 0 & 0740 & \\
\hline 0421 & & 0485 & 3 & 0549 & 4 & 0613 & $R_{2}$ & 0677 & $=$ & 0741 & \\
\hline 0422 & 6 & 0486 & 5 & 0550 & 5 & 0614 & 4 & 0678 & R & 0742 & \\
\hline 0423 & 5 & 0487 & + & 0551 & 2 & 0615 & 5 & 0679 & & 0743 & \\
\hline 0424 & EXC_ & 0488 & 6 & 0552 & $\mathrm{R}$ & 0616 & 1 & 0680 & 7 & 0744 & \\
\hline 0425 & TAN $^{-}$ & 0489 & $=$ & 0553 & & 0617 & EXC & 0681 & 2 & 0745 & \\
\hline 0426 & $L_{B}$ & 0490 & $\mathbf{R}$ & 0554 & 5 & 0618 & $\operatorname{SIN}^{-}$ & 0682 & CLDP & 0746 & \\
\hline 0427 & SIN & 0491. & 4 & 0.5 .5 .5 & 0 & ก 1.9 & T,RT, & ก 683 & $=$ & $07 \triangle 7$ & D \\
\hline 0428 & & 0492 & 3 & 0556 & + & 0620 & 4 & 0684 & $K_{-}$ & 0748 & A \\
\hline 0429 & 3 & 0493 & 5 & 0557 & ( & 0621 & $\mathbf{R}$ & 0685 & $2^{-}$ & 0749 & $\pi$ \\
\hline 0430 & 6 & 0494 & $\mathbf{R}$ & 0558 & $\mathrm{R}$ & 0622 & $0^{-}$ & 0686 & C & 0750 & $\mathbf{E}$ \\
\hline 0431 & 5 & 0495 & 4 & 0559 & $\mathrm{R}_{-}$ & 0623 & 0 & 0687 & o & 0751 & PAPR \\
\hline 0432 & + & 0496 & 3 & 0560 & 4 & 0624 & 0 & 0688 & $\mathrm{R}$ & 0752 & PAPR \\
\hline 0433 & 1 & 0497 & 6 & 0561 & 3 & 0625 & + & 0689 & $\mathrm{R}$ & 0753 & PAPR \\
\hline 0434 & $=$ & 0498 & + & 0562 & 1 & 0626 & 1 & 0690 & $=$ & 0754 & PAPR \\
\hline 0435 & & 0499 & 6 & 0563 & + & 0627 & $=$ & 0691 & $=$ & 0755 & PAPR \\
\hline 0436 & & 0500 & $=$ & 0564 & R & 0628 & $\mathbf{R}$ & 0692 & $\mathbf{R}_{-}$ & 0756 & D \\
\hline 0437 & 6 & 0501 & $\mathbf{R}$ & 0565 & $\mathrm{R}$ & 0629 & 0 & 0693 & & 0757 & \\
\hline 0438 & 5 & 0502 & 4 & 0566 & & 0630 & 0 & 0694 & 5 & 0758 & $\bar{S}$ \\
\hline 0439 & & 0503 & 3 & 0567 & 3 & 0631 & 0 & 0695 & 3 & 0759 & $\mathrm{P}$ \\
\hline 0440 & & 0504 & 6 & 0568 & 2 & 0632 & R AD & 0696 & - & 0760 & C \\
\hline 0441 & 3 & 0505 & EXC & 0569 & ) & 0633 & GODP & 0697 & 0 & 0761 & \\
\hline 0442 & 1 & 0506 & & 0570 & 1 & 0634 & LBL & 0698 & ) & 0762 & 2 \\
\hline 0443 & + & 0507 & LBL & 0571 & 2 & 0635 & 5 & 0699 & $I F=0$ & 0763 & $\mathrm{~T}$ \\
\hline 0444 & 6 & 0508 & TAN $^{-}$ & 0572 & $=$ & 0636 & & 0700 & 1 & 0764 & H \\
\hline 0445 & $=$ & 0509 & $\mathrm{R}$ & 0573 & R & 0637 & 4 & 0701 & $=$ & 0765 & E \\
\hline
\end{tabular}




\begin{tabular}{|c|c|c|c|c|c|c|c|c|c|c|c|}
\hline 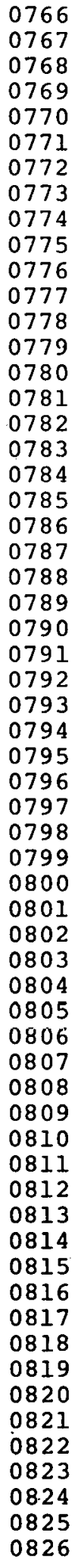 & 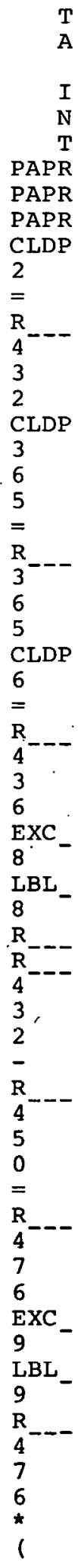 & $\begin{array}{l}0827 \\
0828 \\
0829 \\
0830 \\
0831 \\
0832 \\
0833 \\
0834 \\
0835 \\
0836 \\
0837 \\
0838 \\
0839 \\
0840 \\
0841 \\
0842 \\
0843 \\
0844 \\
0845 \\
0846 \\
0847 \\
0848 \\
0849 \\
0850 \\
0851 \\
0852 \\
0853 \\
0854 \\
0855 \\
0856 \\
0857 \\
0858 \\
0859 \\
0860 \\
08885 \\
0861 \\
0887 \\
0887 \\
0862 \\
0863 \\
0887 \\
0864 \\
0865 \\
0867 \\
0875 \\
0867 \\
0868 \\
0869 \\
0870 \\
0871 \\
0873\end{array}$ & 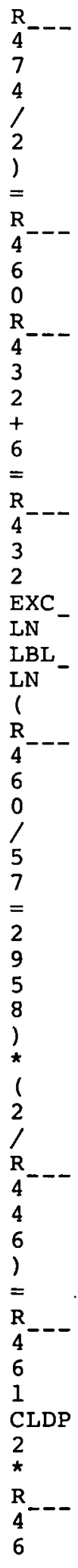 & $\begin{array}{l}0888 \\
0889 \\
0890 \\
0891 \\
0892 \\
0893 \\
0894 \\
0895 \\
0896 \\
0897 \\
0898 \\
0899 \\
0900 \\
0901 \\
0902 \\
0903 \\
0904 \\
0905 \\
0906 \\
0907 \\
0908 \\
0909 \\
0910 \\
0911 \\
0912 \\
0913 \\
0914 \\
0915 \\
0916 \\
0917 \\
0918 \\
0919 \\
0920 \\
0921 \\
0922 \\
0923 \\
0924 \\
0925 \\
0926 \\
0994 \\
0947 \\
094 \\
0928 \\
094\end{array}$ & 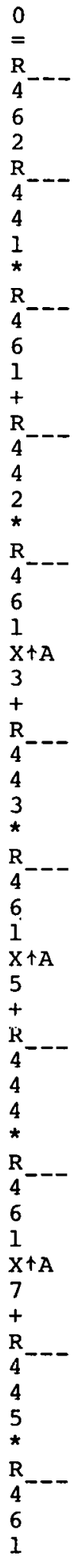 & $\begin{array}{l}0949 \\
0950 \\
0951 \\
0952 \\
0953 \\
0954 \\
0955 \\
0956 \\
0957 \\
0958 \\
0959 \\
0960 \\
0961 \\
0962 \\
0963 \\
0964 \\
0965 \\
0966 \\
0967 \\
0968 \\
0969 \\
0970 \\
0971 \\
0972 \\
0973 \\
0974 \\
0975 \\
0976 \\
0977 \\
0978 \\
0979 \\
0980 \\
0981 \\
0982 \\
0983 \\
0984 \\
0985 \\
0986 \\
0987 \\
0988 \\
0989 \\
0990 \\
0991 \\
0992 \\
0993 \\
0994 \\
0995 \\
0996 \\
0997 \\
0998 \\
0999 \\
1000 \\
1001 \\
1002 \\
1003 \\
1004 \\
1005 \\
1006 \\
1007 \\
1008 \\
1009\end{array}$ & 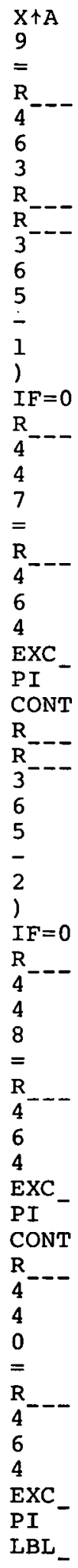 & $\begin{array}{l}1010 \\
1011 \\
1012 \\
1013 \\
1014 \\
1015 \\
1016 \\
1017 \\
1018 \\
1019 \\
1020 \\
1021 \\
1022 \\
1023 \\
1024 \\
1025 \\
1026 \\
1027 \\
1028 \\
1029 \\
1030 \\
1031 \\
1032 \\
1033 \\
1034 \\
1035 \\
1036 \\
1037 \\
1038 \\
1039 \\
1040 \\
1041 \\
1042 \\
1043 \\
1044 \\
1045 \\
1046 \\
1047 \\
1048 \\
1049 \\
1050 \\
1051 \\
1052 \\
1053 \\
1054 \\
1055 \\
1056 \\
1057 \\
1058 \\
1059 \\
1060 \\
1061 \\
1062 \\
1063 \\
1064 \\
1065 \\
1066 \\
1067 \\
1068 \\
1069 \\
1070\end{array}$ & 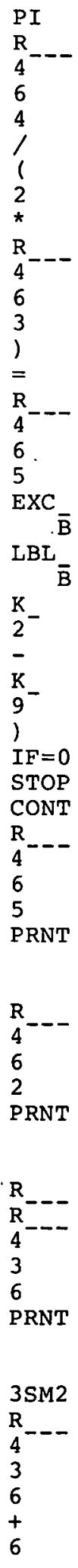 & $\begin{array}{l}1071 \\
1072 \\
1073 \\
1074 \\
1075 \\
1076 \\
1077 \\
1078 \\
1079 \\
1080 \\
1081 \\
1082 \\
1083 \\
1084 \\
1085 \\
1086 \\
1087 \\
1088\end{array}$ & $\begin{array}{l}= \\
\mathrm{R}_{---} \\
4 \\
3 \\
6 \\
\mathrm{R}^{-}--- \\
3 \\
6 \\
5 \\
+ \\
1 \\
= \\
\mathrm{R} \\
3 \\
6 \\
5 \\
\text { EXC_- } \\
8\end{array}$ \\
\hline . & & & & & & & & & & & \\
\hline
\end{tabular}




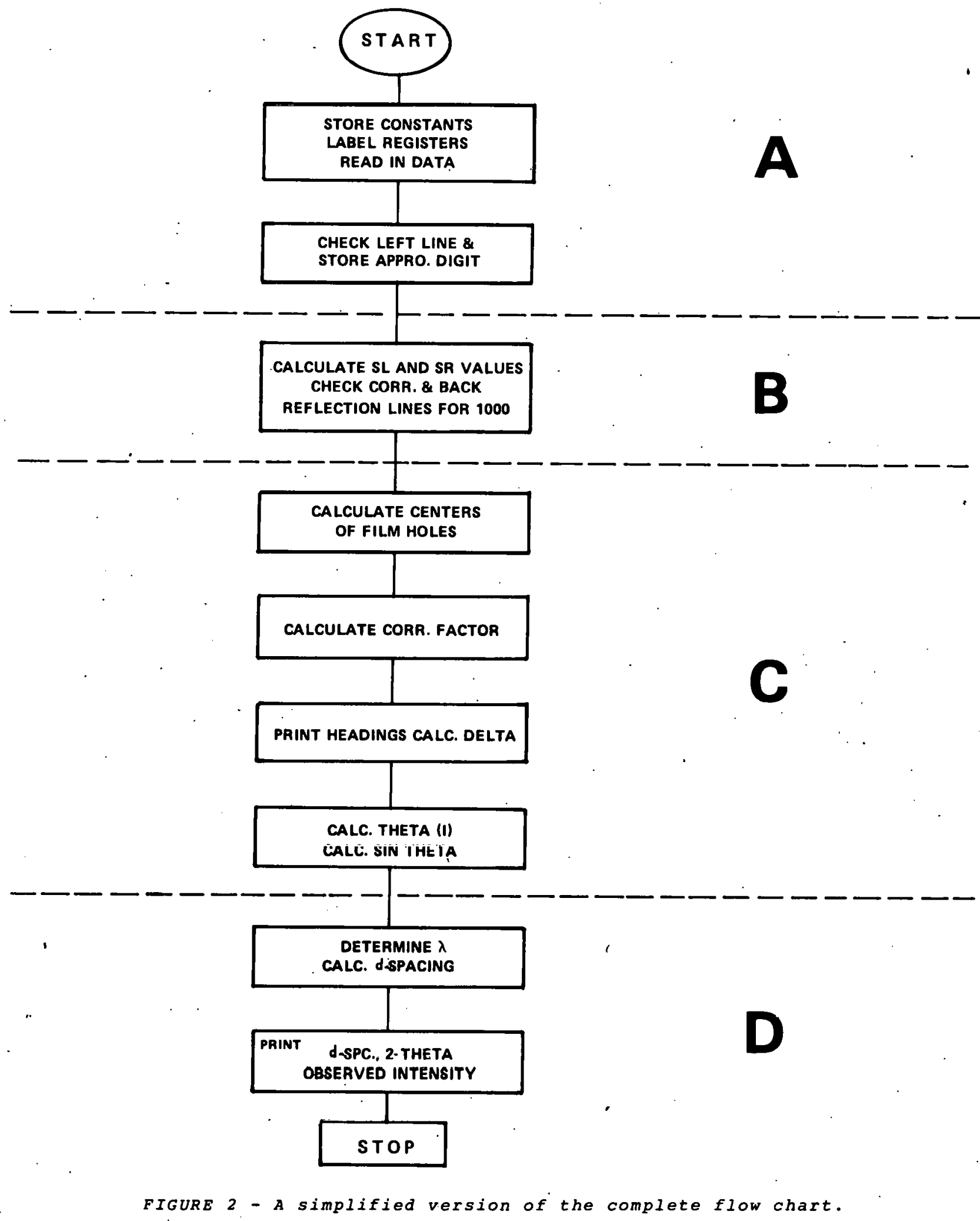




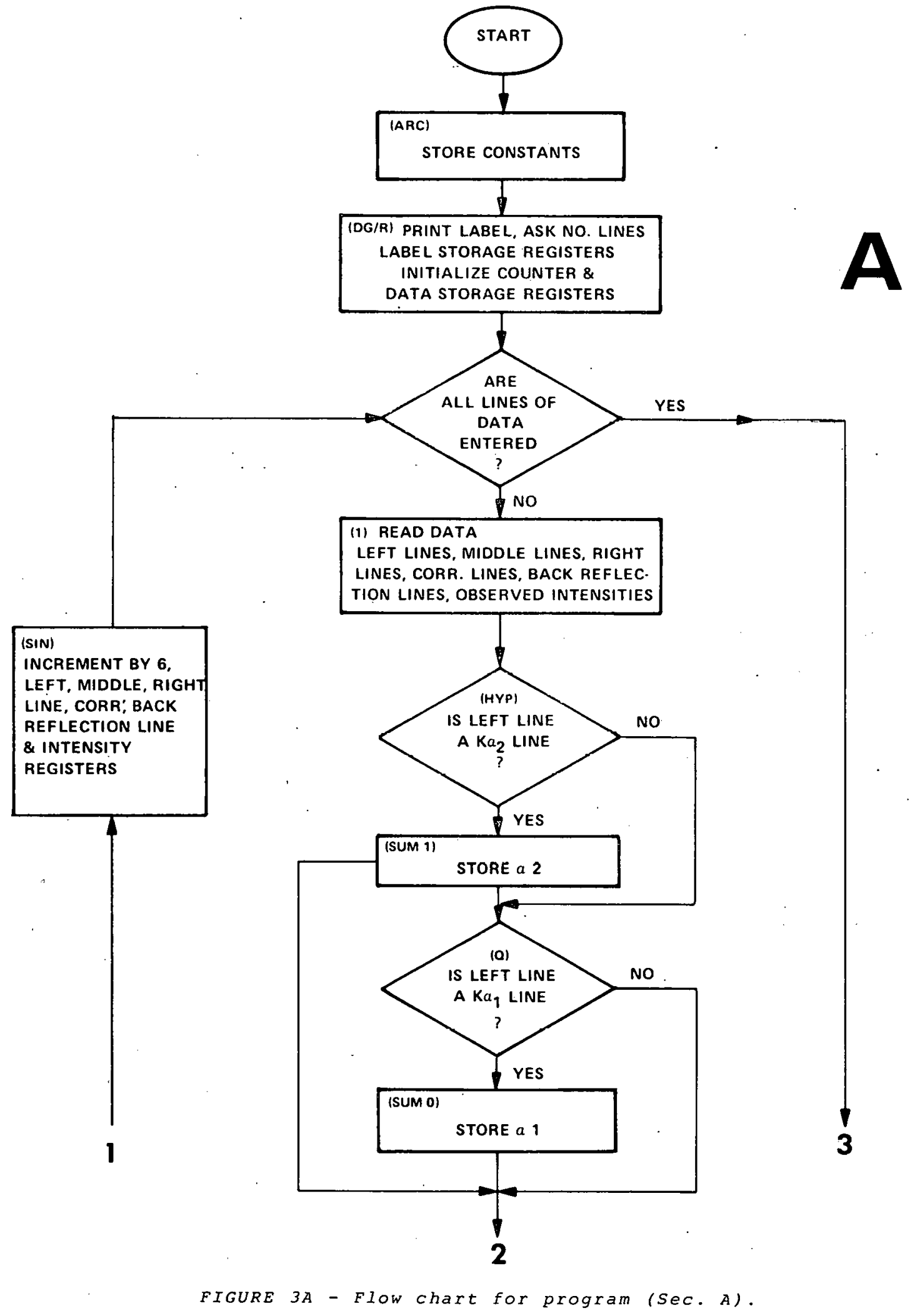

LEFT, MIDDLE, RIGH

LINE, CORR', BACK

(a)

IS LEFT LINE

A $K a_{1}$ LINE 
$\downarrow$

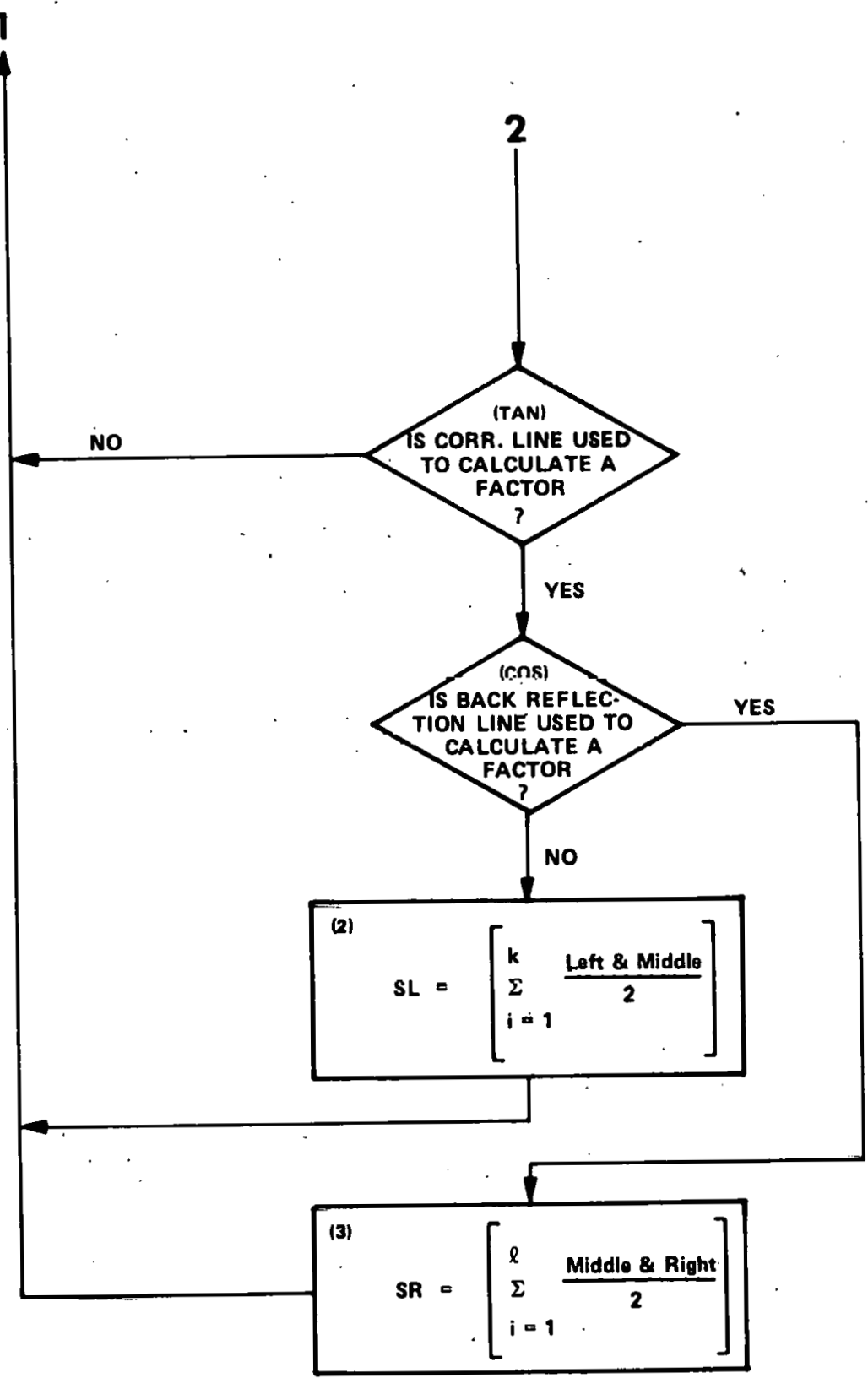




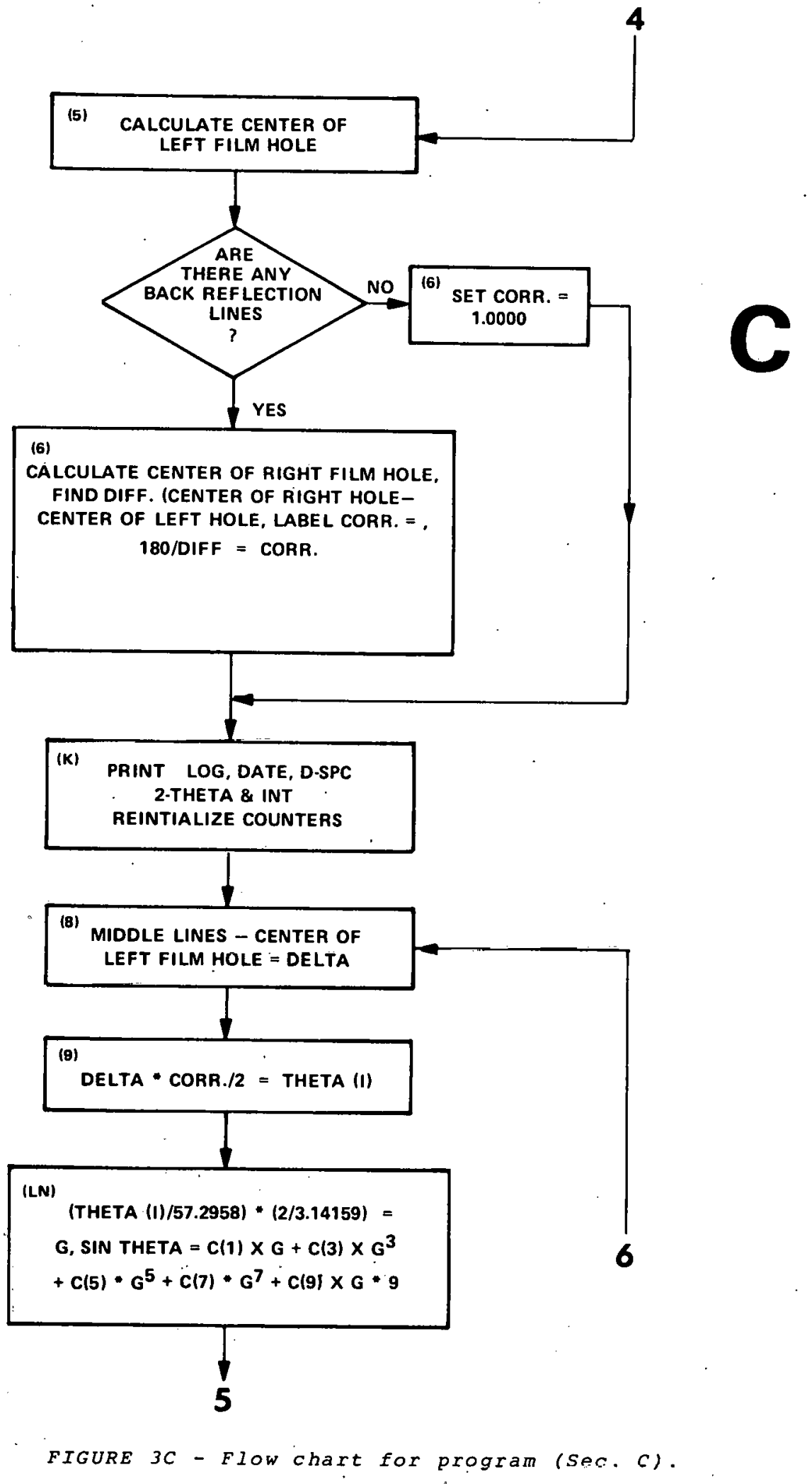




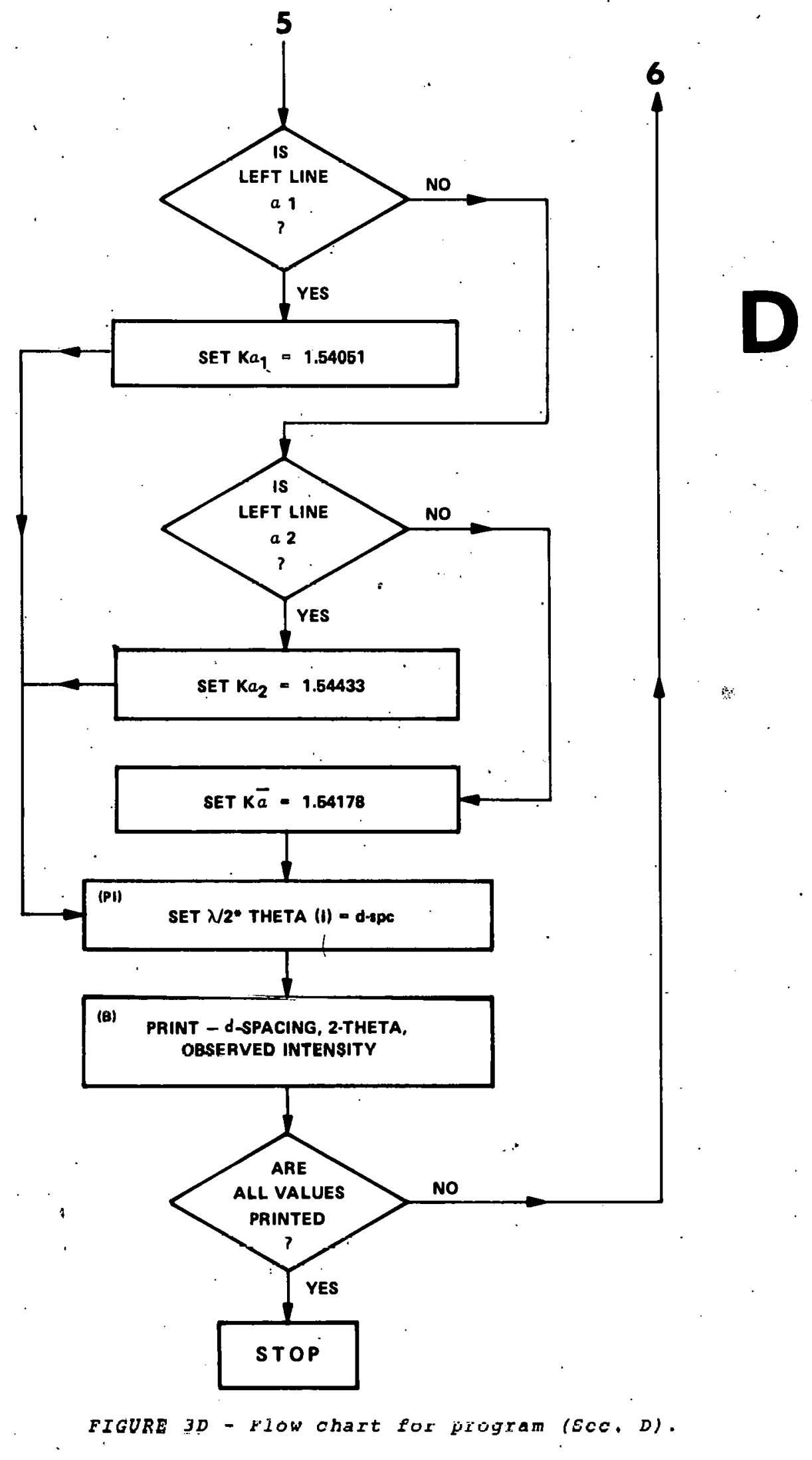


which is used to determine whether the data calculated is based on copper $K \bar{\alpha}$, $\mathrm{K} \alpha$, or $\mathrm{K} \alpha_{2}$ radiation. There are 30 more $\mathrm{R}$ registers reserved for decision màking and constant value storage. Since the data computed is immediately printed and not stored, an excess of 150-plus storage registers remain for the expansion of the program.

The line measurements are collected in the usual manner. The program begins by typing out the label and then asks the user for the number of sets of data to be entered $\mathrm{N}$, followed by an equal sign $(=)$. The user answers the question by striking the appropriate numeral/s key, for example, 37. Depressing the continue (CONT) button causes the paper to advance and the calculator to respond with a typed symbol for the left line meassurement, for example, $L$ (I). Each user input is preceded by a symbol: left line $L(I)$, middle line $M(I)$, right line $R(I)$. See Figure 4. A one (1) entered after $C(I)$ indicates that the data in that input set will be used in computing the film correction factor. A one (1) following $B(I)$ indicates that the line is a back reflection line. A one (1) entered after $L(I)$ indicates the line to be a $K \alpha$, line. A two (2) following $L(I)$ indicates the line is a $\mathrm{K} \alpha 2$. A value greater than 2 , or a zero $(0)$ entered after $L(I)$ indicates the line is a $K \bar{\alpha}$ (av). Values entered after $I(I)$ are the observed intensities of each line and are based on a scale from 10 to 1 with 10 being the most intense and 1 indicating the line is barely visible.

When all of the line measurements and symbols (for example, 0,1, and 2 ) have been entered, the data are completed and printed line by line. The printed data from a typical powder pattern are shown in Figures $5 \mathrm{a}$ and $5 \mathrm{~b}$. The paper tape output serves as a permanent record for the user.
Program to CALC D Spacings Enter $\mathrm{N}=10$

\begin{tabular}{|c|c|c|c|c|c|}
\hline$L(I)$ & 76.3 & $R(I)$ & 0 & $B(I)$ & 0 \\
\hline$M(I)$ & 151. & $C(I)$ & 0 & $I(I)$ & 1. \\
\hline$R(I)$ & 0 & $B(I)$ & 0 & $L(I)$ & 0. \\
\hline$C(I)$ & 1. & $I(I)$ & 2 . & $M(I)$ & 225.2 \\
\hline$B(I)$ & 0 & $L(I)$ & 0. & $R(I)$ & 362.9 \\
\hline$I(I)$ & 9. & $M(I)$ & 193.3 & $C(I)$ & 1. \\
\hline$L(I)$ & 70.2 & $R(I)$ & 0. & $B(I)$ & 1. \\
\hline$M(I)$ & 157. & $C(I)$ & 0. & $I(I)$ & 2. \\
\hline$R(I)$ & 0 . & $B(I)$ & 0 & $L(I)$ & 0 \\
\hline$C(I)$ & 1. & $I(I)$ & 1. & $M(I)$ & 243.5 \\
\hline$B(I)$ & 0. & $L(I)$ & 0 . & $R(I)$ & 344.7 \\
\hline$I(I)$ & 10. & $M(I)$ & 209 & $C(I)$ & 1. \\
\hline$L(I)$ & 50.7 & $R(I)$ & 0 & $B(I)$ & 1. \\
\hline$M(I)$ & 176.7 & $C(I)$ & 0 & $I(I)$ & 2. \\
\hline$R(I)$ & 0 & $B(I)$ & 0 & $L(I)$ & 0 \\
\hline$C(I)$ & 1. & $I(I)$ & 1. & $M(I)$ & 261.2 \\
\hline$B(I)$ & 0 & $L(I)$ & 0 & $R(I)$ & 327.1 \\
\hline$I(I)$ & 6. & $M(I)$ & 220.9 & $C(I)$ & 1. \\
\hline$L(I)$ & 0. & $R(I)$ & 0 & $B(I)$ & 1. \\
\hline$M(I)$ & 189.3 & $C(I)$ & 0. & $I(I)$ & 1. \\
\hline
\end{tabular}

FIGURE 4 - Sample input data for Tektronix-31 d-spacing program.

Corr. =

.9975062344

Log Date

D_Spc 2Theta Int

$\begin{array}{cc}2.413332908 & 62.89276808 \\ 37.25685786 & 6 . j \\ 9 . & 1.259725815 \\ 2.092168702 & 75.46134663 \\ 43.24189526 & 2 . \\ 10 . & 1.205194215 \\ 1.477648404 & 79.45137157\end{array}$

111.2718204

.8522337398

1.04379278

1 .

.9583072861

106.9825436

1 .

.9338830885
129.5261845

2 .

.8036222561

147.1820449

FIGURE $5 a$ - Output data from program used to process d-spacings when a camera radius correction is computed (that is, when back reflection lines are available). 


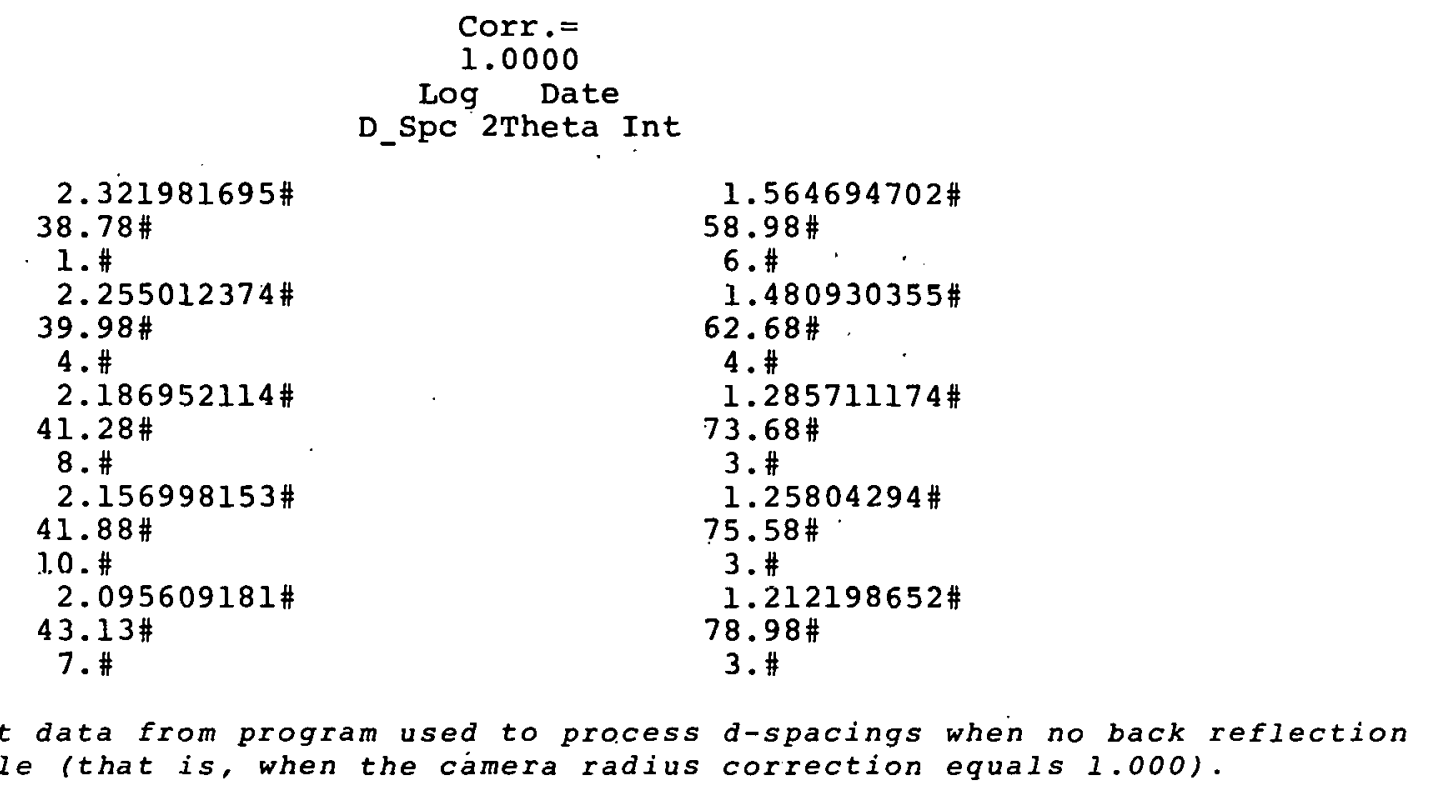

\section{Conclusions}

The program described in this report represents the initial attempt by the $x$-ray diffraction laboratory personnel to provide $X-r a y$ data computation backup to the PDP-8/I computer. The Tektronix31 calculator has shown that it is an accurate, relatively rapid, and satisfactory tool - able to provide the necessary data computations in the event of a temporary loss of the PDP-8/I computer services.

\section{Glossary of Program Terms}

N Number of lines read from $X-r a y$ powder diffraction pattern.

PI $\quad 3.14159$

SL Sum left, which is

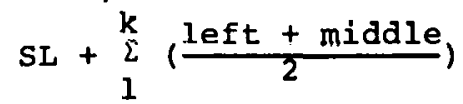

SR Sum right, which is

$$
\mathrm{SR}+\sum_{1}^{\ell}\left(\frac{\text { middle }+ \text { right }}{2}\right)
$$

Left $=$ lines which are located to the left of the hole at the left side of the film punched for. standard Wilson or strausman's load cameras.

Middle $=$ lines which are located between the left and right holes on the film.
Right $=$ lines located to the right of the hole at the right side of the film.

L Counter used to determine SL average.

R Counter used to determine SK average.

Corr. Camera correction factor which adjusts data for film expansion or shrinkage.

DELTA Middle line minus SL average. 


\section{Subroutines Used}

$\begin{array}{llll}\text { 1) } & \text { DG/R } & \text { 11) } & 3 \\ \text { 2) } & \text { I } & 12) & 5 \\ \text { 3) } & \text { SIN } & 13) & 6 \\ \text { 4) } & \text { HYP } & 14) & \text { K } \\ \text { 5) } & \text { SUM O } & 15) & 8 \\ \text { 6) } & \text { SUM 1 } & 16) & 9 \\ \text { 7) } & \text { Q } & 17) & \text { LN } \\ \text { 8) } & \text { TAN } & 18) & \text { PI } \\ \text { 9) } & \text { COS } & 19) & \text { B }\end{array}$

NOTE: All subroutines are appropriately labeled and defined on the flow chart which appears in Figure 2 . 


\section{References}

1. P. W. Seabaugh and R. R. Eckstein, Program for Calculating $X$-Ray Diffraction Interplanar (d) spacings with a PDP-8/I Computer, MLM-1646 (Oct. 15, 1969).

2. Tektronix-31 Operators Manual, Tektronix, Inc., Beaverton, Oregon (1973). 


\section{Distribution}

\section{EXTERNAL}

TIC, UC-4 (189)

J. A. Chacon, DAO

R. K. Flitcraft, MRC

Technical Report Librày, Monsanto, St. Louis

\section{CONSULTANTS}

W. E. Moddeman

University of Dayton Research Institute

D. White

University of Pennsylvania

\section{INTERNAL}
T. C. Bishop
D. R. Bohl
W. T. Cave
R. R. Eckstein
Y. G. Ishida
C. W. Huntington
J. R. McClain
W. D. Pardieck
D. R. Sellers
R. E. Vallee
Document Control
Library (15)
Publications (15) 